\title{
Drug Induce Liver Injury is Associated with High Mortality - A Study from a Tertiary Care Hospital in Pakistan
}

\author{
Adeel Abid ${ }^{1}$, Faryal Subhani ${ }^{1}$, Farhana Kayani ${ }^{1}$, Safia Awan ${ }^{2}$ and Shahab Abid ${ }^{2}$ \\ ${ }^{1}$ Medical College, Aga Khan University, Pakistan \\ ${ }^{1}$ Section of Gastroenterology and Department of Medicine, Aga Khan University, Pakistan
}

Submission: December 03, 2019; Published: December 10, 2019

*Corresponding author: Shahab Abid, Professor and Head Section of Gastroenterology, Department of Medicine, Aga Khan University, Stadium Road P.O Box 3500, Karachi, Pakistan

\begin{abstract}
Background and Aim: In light of the paucity of data on drug induced liver injury DILI especially from South Asia, this study aims to evaluate the clinical spectrum and predictors of mortality and morbidity of hospitalized patients with suspected DILI.

Patients and Methods: DILI cases were identified and categorized on basis of COIMS/RUCAM score and the exclusion of other liver diseases. Clinical and laboratory parameters were analyzed to identify the predictors of morbidity (prolonged hospital stay. $>5$ days) and mortality.

Results: Out of 462 patients, there were 264 (57.6\%) males and the mean of the cohort was 50.83 years (range, 20-94 years). DILI was classified as definite or highly probable in $(31.1 \%$, probable in $62.5 \%$ and possible in $7.4 \%$ of cases. Pattern of liver injury was hepatocellular in $25.1 \%$, cholestatic in $56.17 \%$ and mixed in $18.72 \%$ of patients. Anti-tuberculous drugs (ATDs) were found to be the most common cause of DILI in 295 (63.9\%) encephalopathy was present in 21.6\% patients other presenting symptoms were abdominal pain (57.1\%), vomiting $(57.1 \%)$, jaundice $(54.1 \%$ ) and pruritus $(42.3 \%)$. In-hospital mortality was $26.5 \%$ and prolonged hospital stay was observed in $35.93 \%$ of patients. Mortality was significantly greater in patients with encephalopathy, male gender, hepatocellular pattern of DILI, increased INR and use of ventilator support.

Conclusion: The most frequent cause of DILI was ATDs in hospitalized patients in our study. More than a quarter of patients died during hospital stay. A close control of clinical and biochemical parameters is required especially ATDs in our region.

Keywords: Drug induced liver injury; Spectrum and predictors of outcome; Liver dysfunction; Liver tests; Liver injury; Acute liver failures; Autoimmune hepatitis; Anti-inflammatory drugs; Hepatotoxicity; Hepatitis B virus

Abbrevations: DILI: Drug Induced Liver Injury; ALT: Alanine Aminotransferase; AST: Aspartate Aminotransferase; ALP: Alkaline Phosphatase; ULN: Upper Limit Of Normal; FDA: Food and Drug Administration; LFTs: Liver Function Tests; RUCAM: Roussel Uclaf Causality Assessment Method; NSAIDs: non-Steroidal Anti-Inflammatory Drugs; HDS: Herbal And Dietary Supplements; MTAs: Molecular Targeted Agents; TKI: Tyrosine Kinase Inhibitor; EGFR: Epidermal Growth Factor Receptor; HBV: Hepatitis B Virus; HCV: Hepatitis C Virus; ATDs: Anti-Tuberculous Drugs
\end{abstract}

\section{Introduction}

Drug induced liver injury (DILI) is defined as hepatotoxicity caused by various medications, herbs, or other xenobiotics, subsequently leading to abnormalities in liver tests or liver dysfunction with the reasonable exclusion of other etiologies [1]. Specific laboratory criteria are utilized to identify DILI: typically, a 3-5 times elevation of liver enzymes, namely transaminases (alanine aminotransferase [ALT] or aspartate aminotransferase

[AST]), alkaline phosphatase (ALP), or bilirubin, above their upper limit of normal (ULN) is required [2]. Altogether, in excess of a thousand medicines and chemicals have been implicated in drug induced liver injury $[3,4]$.

In the United States, DILI accounts for nearly $10 \%$ of the total cases of acute hepatitis, $5 \%$ of all hospital admissions, $50 \%$ 
of all cases of acute liver failures [5]. DILI carries a mortality rate of approximately $10 \%$ [3-5]. It is the premier reason for drug withdrawal by the Food and Drug Administration (FDA) in the United States [5,6].

The wide spectrum of clinical symptomatology, nonavailability of specific diagnostic markers and lack of standardization between studies performed to date make it difficult to establish causality to a particular drug. Moreover, causal association to a specific drug is heterogeneous. It depends on the exclusion of other causes notably viral and autoimmune hepatitis. Causality is dependent on the temporal relationship of the drug to the derangement in patient's liver function tests (LFTs). As a result, sometimes certain scoring system such as Roussel Uclaf Causality Assessment Method (RUCAM) [7] is used to assess the probability of association. The RUCAM system is a means of assigning points for clinical, biochemical, serologic and radiologic features of liver injury which gives an overall assessment score which reflects the likelihood that the hepatic injury is due to a specific medication [8].

Annual incidence of DILI ranges from 1.3 to 19 per 100,000 in various databases, depending on the country of origin, type of data and method of obtaining information [9-11] .The largest drug category responsible for DILI is antimicrobials, lead by amoxicillin-clavulanate [12,13]. Amongst antibiotics, ATDs are another major group associated with DILI especially in the developing world. Approximately $5.3 \%$ of all the cases in the United States DILI Network (US DILIN) were reported due to isoniazid (second only to amoxicillin-clavulanate) likewise 7\% of the cases in the Spanish DILI Registry were due to isoniazid alone or in combination with other drugs $[12,13]$.0ther common drug groups include non-steroidal anti-inflammatory drugs (NSAIDs) [9,13], herbal and dietary supplements (HDS) [14] and rarely statins $[12,15,16]$.

A growing concern for pharmaceutical industry regarding drug development is hepatotoxicity induced by the newer molecular targeted agents (MTAs) which are increasingly being used in oncology. A third of patients treated with a protein kinase inhibitor experience liver injury, with pazopanib, sunitinib and regorafenib identified as the potentially lethal agents [17]. Similarly, $10 \%$ of patients treated with immune checkpoint inhibitors, such as ipilimumab, are susceptible to DILI [17]. Additionally, the epidermal growth factor receptor (EGFR) tyrosine kinase inhibitor (TKI) gefitinib is associated with $18.5 \%$ frequency of hepatotoxicity. It has resulted in casualties as well [18].

In many countries, DILI registries have been set up which record every DILI case with a formal causality determination process, providing in-depth information about the types of drugs that cause DILI, the pattern of injury and the risk of mortality and morbidity. There is paucity of data from South Asia with no such DILI registries in place. This study aims to provide an analysis of clinical presentation and outcome of patients admitted with the discharge diagnosis of DILI from Pakistan.

\section{Patients and Methods}

\section{Study design}

A retrospective cross-sectional study.

\section{Study setting and population}

Patients admitted at Aga Khan University Hospital Karachi Pakistan, from January 2010 through December 2016, and discharged with a diagnosis of DILI, were recruited. The course of their hospital stay was reviewed through the medical record system.

\section{Inclusion criteria}

Patients with suspected diagnosis of DILI with clear documentation of the possible drug implicated were included.

\section{Exclusion criteria}

Patients with known or suspected acetaminophen toxicity, history of bone marrow or liver transplantation before the liver injury event, history of malignancy of liver and metastasis to liver, underlying hepatitis C virus (HCV), hepatitis B virus (HBV), or nonalcoholic fatty liver disease were all excluded alongside cases with other types of underlying chronic liver disease.

\section{Criteria for diagnosis of DILI}

The diagnosis of DILI and the causal relationship between liver injury event and implicated drugs were evaluated in a formal and standardized fashion by using a causality instrument: Roussel Uclaf Causality Assessment Method (RUCAM) [8]. Points were awarded for seven components comprising of the following: time to onset of the injury following start of the drug, subsequent course of the injury after stopping the drug, specific risk factors (age, alcohol use, pregnancy), use of other medications with a potential for liver injury, exclusion of other causes of liver disease, known potential for hepatotoxicity of the implicated drug and response to re-challenge. The RUCAM provides a semi-quantitative evaluation of causality by assigning -3 to +3 points to each of the aforementioned seven components. Based on the final score, a causal relationship between the implicated agent and the liver injury event was categorized as highly probable (>8), probable (6-8), possible (3-5), unlikely (1 or 2$)$, or excluded $(<0)$.

\section{Criteria for assessment of clinical patterns of liver injury}

According to the Council for International Organizations of Medical Sciences (CIOMS) criteria, DILI is classified as hepatocellular, cholestatic or mixed based on its R-value [8]. The $\mathrm{R}$-value is defined as the serum ALT/ULN divided by the serum ALP/ULN ratio. R-values $>5$ were classified as hepatocellular, < 2 as cholestatic and 2-5 as mixed injury. 


\section{Criteria for severity assessment}

The severity assessment was done according to the Chinese guidelines for the diagnosis and treatment of DILI in 2015 [19]. The severity was scored as follows:

a) Mild: serum enzyme elevations with total bilirubin $(\mathrm{TBil})<2.5 \times$ ULN and International Normalization Ratio $($ INR $)<1.5$.

b) Moderate: serum enzyme elevations and TBil $\geq 2.5 \times$ ULN or an INR $\geq 1.5$.

c) Severe: serum enzyme elevations and TBil $\geq 5 \times$ ULN with or without an INR $\geq 1.5$.

d) 4. Acute liver failure: serum enzyme elevations and TBil $\geq 10 \times$ ULN or a daily elevation of TBil $\geq 17.1 \mu \mathrm{mol} / \mathrm{L}$, an INR $\geq 2.0$ and signs of hepatic or other organ failure related to DILI.

\section{Assessment of patient morbidity and mortality}

In-hospital morbidity was quantified in terms of prolonged hospital stay, defined as hospital stay for more than 5 days. Predictors of mortality and morbidity were assessed by considering patients' clinical and laboratory parameters including liver synthetic functions (prothrombin time and serum albumin).

\section{Statistical analysis}

The statistical analysis was conducted by using the Statistical Package for Social Science (SPSS) (Release 19.0 standard version, copyright (C) SPSS). A descriptive analysis was performed and results are presented as mean \pm standard deviation for quantitative variables and numbers (percentages) for qualitative variables. To analyze the risk factors for poor outcome, the categorical variables were evaluated using the chisquare test while the means were compared by Student t-test. Factors predicting prolonged hospital stay were analyzed by multivariate logistic regression analysis. To establish statistical significance, $\mathrm{p}$ value $<0.05$ was considered significant.

\section{Results}

A total of 462 DILI cases were identified (Figure 1), out of which $264(57.6 \%)$ patients were male with a mean age of 50.83 (range: 20-94). By using the RUCAM model for drug causality assessment, DILI was classified as definite or highly probable in $141(31.1 \%)$, probable in $289(62.5 \%)$ and possible in $34(7.4 \%)$ cases.

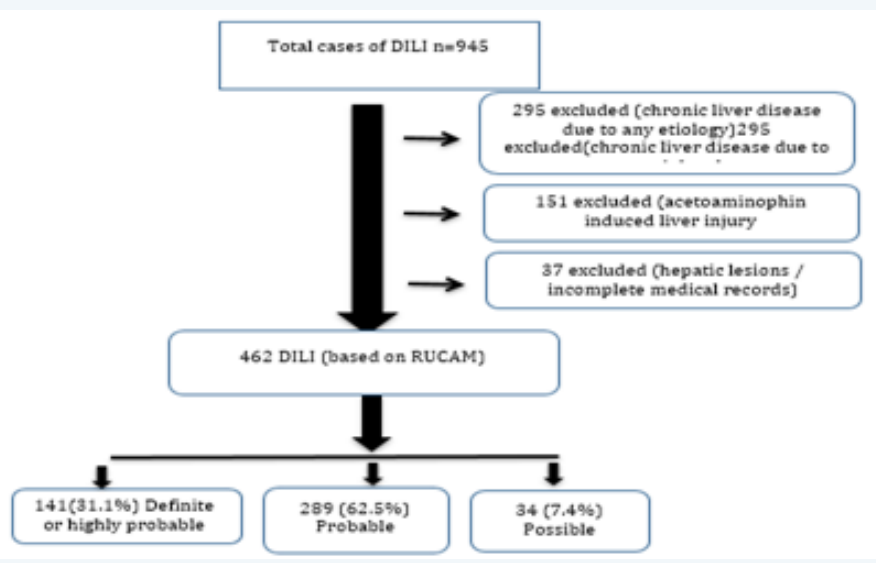

Figure 1: Flow diagram showing selection of DILI cases.

\section{Pattern of liver injury}




\section{Advanced Research in Gastroenterology \& Hepatology}

Pattern of liver injury was hepatocellular in 116 (25.1\%), with a discharge diagnosis of DILI (Figure 2). cholestatic in $260(56.17 \%)$ and mixed in $86(8.72 \%)$ patients

\section{Severity of liver injury}

Table 1: Severity of DILI $\Omega$ and relationship of age and gender with mortality $(n=462)$.

\begin{tabular}{|c|c|c|c|c|c|}
\hline & Mild; $n=204(44.2 \%)$ & Moderate; $n=78(16.9 \%)$ & Severe; $n=64(13.9 \%)$ & ACLF; $n=116(25.1 \%)$ & p Value \\
\hline \multicolumn{6}{|l|}{ Mortality } \\
\hline Yes & $42(20.6)$ & $20(25.6)$ & $16(25)$ & $44(38.6)$ & 0.006 \\
\hline No & $162(79.4)$ & $58(74.4)$ & $48(75)$ & $70(61.4)$ & \\
\hline \multicolumn{6}{|l|}{ Age } \\
\hline$\leq 35$ years & $46(22.5)$ & $16(20.5)$ & $12(18.8)$ & $28(24.1)$ & 0.19 \\
\hline $36-45$ & $36(17.6)$ & $14(17.9)$ & $8(12.5)$ & $20(17.2)$ & \\
\hline $46-55$ & $40(19.6)$ & $10(12.8)$ & $12(18.8)$ & $26(22.4)$ & \\
\hline $55-65$ & $40(19.6)$ & $18(23.1)$ & $12(18.8)$ & $30(25.9)$ & \\
\hline$>65 \mathrm{yrs}$ & $42(20.6)$ & $20(25.6)$ & $20(31.3)$ & $12(10.3)$ & \\
\hline \multicolumn{6}{|l|}{ Gender } \\
\hline Male & $102(50)$ & $52(66.7)$ & $50(78.1)$ & $62(53.4)$ & $<0.001$ \\
\hline Female & $102(50)$ & $26(33.5)$ & $14(21.9)$ & $54(46.6)$ & \\
\hline
\end{tabular}

DILIS: Drug Induced Liver Injury

The severity of liver injury was found to be mild in 204 (44\%), moderate in $78(16.8 \%)$, and severe in $54(13.8 \%)$ patients while $116(25.1 \%)$ cases were seen to have had liver failure due to drug intake. Mortality was significantly high in patients with liver failure ( $p$ value $=0.006$ ) (Table 1 ).

\section{Presenting features of patients with DILI}

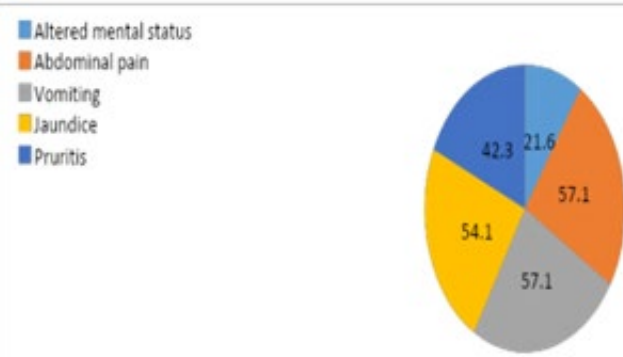

Figure 3: Presenting features of patients with drug induced liver injury $(n=462)$.

Encephalopathy (encephalopathy) was present in 98 $(21.6 \%)$ patients on the day of hospital admission while patients who presented with abdominal pain, vomiting, jaundice and pruritus were in the following order: $57.1 \%, 57.1 \%, 54.1 \%$, and

\section{Drug categories causing DILI}

42.3\% (Figure 3). Furthermore, mean total bilirubin levels, ALT and AP levels were $5.37 \mathrm{mg} / \mathrm{dl}$ (range: $0.20-79.1$ ), 358.65 (range: 7-8938) IU/L and 168.68 (range: 32-1040) IU/L respectively.

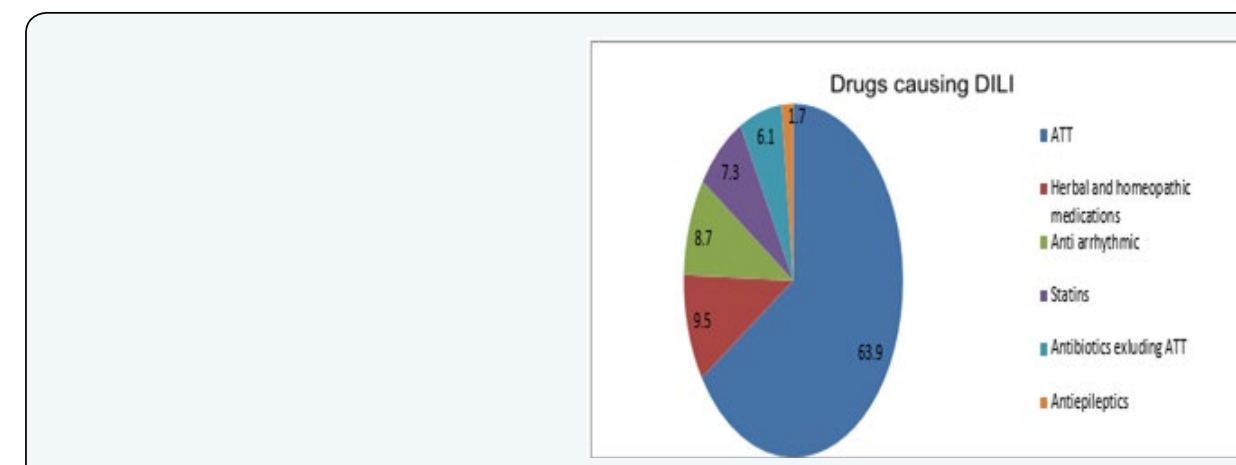

Figure 4: Drug categories causing drug induced liver injury $(n=462)$. 


\section{Advanced Research in Gastroenterology \& Hepatology}

The top three causes of DILI in our study were antituberculous drugs (ATDs) followed by homeopathic or herbal medications and antiarrhythmic drugs. Other drugs implicated are displayed on Figure 4.

\section{Predictors of mortality and morbidity}

In-hospital mortality was 122 out of 462 (26.5\%) and morbidity (quantified as prolonged hospital stay more than 5 days) was observed in 214 out of 462 (35.93\%) patients. None of the patients underwent a liver transplant due to nonavailability of the facility at our institution and in the city during that time period. On multivariate analysis, mortality was significantly greater in patients with encephalopathy, male gender, hepatocellular pattern of DILI, increased INR ( $>1.5)$, acute liver failure and patients who were on ventilator support in ICU (Table 2). Likewise, prolonged hospital stay (duration of $>5$ days) was associated with female gender, increased ALT, AST aspartate aminotransferase levels, use of ventilator support and mixed pattern of DILI, (Table 3).

Table 2: Predictors of mortality of drug induced liver injury $(n=462)$.

\begin{tabular}{|c|c|c|c|}
\hline & Death; $n=122$ & Survive; $n=340$ & p Value \\
\hline Age, in years & $53.3 \pm 15.1$ & $49.8 \pm 17.0$ & 0.03 \\
\hline \multicolumn{4}{|l|}{ Gender } \\
\hline Male & $80(65.6)$ & $184(54.4)$ & 0.03 \\
\hline Female & $42(34.4)$ & $154(45.6)$ & \\
\hline DM & $30(24.6)$ & $70(20.7)$ & 0.22 \\
\hline Dyslipidemia & $50(41)$ & $108(32)$ & 0.07 \\
\hline ATT & $70(57.4)$ & $224(66.7)$ & 0.08 \\
\hline Antibiotic & $8(6.6)$ & $20(5.9)$ & 0.8 \\
\hline Antiepileptic & 0 & $8(2.4)$ & 0.08 \\
\hline Antifungal & $10(8.2)$ & $16(4.7)$ & 0.15 \\
\hline Lipiget & $10(8.2)$ & $22(6.5)$ & 0.57 \\
\hline Chemotherapy & $10(8.2)$ & $4(1.2)$ & $<0.001$ \\
\hline Herbal & $10(8.2)$ & $32(9.5)$ & 0.63 \\
\hline Anti-malaria & $6(5.6)$ & $4(1.4)$ & 0.02 \\
\hline Digoxin & 0 & $12(4.3)$ & 0.006 \\
\hline Antidepressants & $2(2.0)$ & $14(5.0)$ & 0.17 \\
\hline $\begin{array}{l}\text { Altered Mental } \\
\text { Status }\end{array}$ & $46(37.7)$ & $52(15.4)$ & $<0.001$ \\
\hline Jaundice & $74(60.7)$ & $174(51.5)$ & 0.08 \\
\hline Pruritus & $78(63.9)$ & $184(54.4)$ & 0.06 \\
\hline Abdominal pain & $120(98.4)$ & $326(96.4)$ & 0.26 \\
\hline NAC & $38(31.1)$ & $54(16)$ & $<0.001$ \\
\hline Hospital stay & $10.7 \pm 10.9$ & $6.7 \pm 6.5$ & $<0.001$ \\
\hline Intubation & $32(38.1)$ & $18(7.0)$ & $<0.001$ \\
\hline $\mathrm{TB}$ & $6.9 \pm 12.8$ & $4.8 \pm 7.0$ & 0.09 \\
\hline IB & $2.5 \pm 5.7$ & $1.08 \pm 1.4$ & 0.007 \\
\hline PT & $19.4 \pm 13.0$ & $15.0 \pm 7.0$ & $<0.001$ \\
\hline INR & $1.93 \pm 1.3$ & $1.45 \pm 0.65$ & $<0.001$ \\
\hline $\mathrm{AP}$ & $202.7 \pm 183.1$ & $165.3 \pm 121.2$ & 0.03 \\
\hline
\end{tabular}

Table 3: Predictors of prolong hospital stay $>5$ days of patients with DILI $(n=462)$.

\begin{tabular}{|c|c|c|c|}
\hline Characteristics & $\mathbf{<}$ days; $\mathbf{2 4 8}$ & $\mathbf{> 5}$ days; $\mathbf{2 1 4}$ & $\mathbf{p ~ V a l u e}$ \\
\hline Age, in years & $50.2 \pm 16.8$ & $51.3 \pm 16.4$ & 0.45 \\
\hline Gender & & & \\
\hline Male & $154(62.1)$ & $112(526)$ & 0.03 \\
\hline Female & $94(37.9)$ & $101(47.4)$ & \\
\hline DM & $48(19.4)$ & $31(14.6)$ & 0.17 \\
\hline Dyslipidemia & $51(20.6)$ & $50(23.5)$ & 0.45 \\
\hline ATT & $43(76.8)$ & $37(58.7)$ & 0.03 \\
\hline Antibiotics & $4(7.0)$ & $4(6.3)$ & 0.88 \\
\hline Antiepileptics & $2(3.5)$ & $1(1.6)$ & 0.5 \\
\hline Antifungal & $1(1.8)$ & $3(4.8)$ & 0.35 \\
\hline Amiodarone & $5(8.8)$ & $5(7.9)$ & 0.86 \\
\hline Statins & $6(10.5)$ & $5(7.9)$ & 0.62 \\
\hline Chemotherapy & $1(1.8)$ & $3(4.8)$ & 0.38 \\
\hline Herbal & $4(7.0)$ & $7(11.1)$ & 0.45 \\
\hline Antimalarial & 0 & $2(4.8)$ & 0.49 \\
\hline Mortality & $27(22.1)$ & $34(32.7)$ & 0.07 \\
\hline NAC & $21(17.4)$ & $26(25)$ & 0.16 \\
\hline H/O Alcohol & $1(1.8)$ & $2(3.3)$ & 0.58 \\
\hline Intubation & $7(12.5)$ & $15(25)$ & 0.08 \\
\hline TB & $4.9 \pm 9.2$ & $6.0 \pm 11.4$ & 0.25 \\
\hline DB & $2.6 \pm 2.3$ & $5.1 \pm 8.6$ & 0.03 \\
\hline IB & $1.11 \pm 1.2$ & $2.72 \pm 5.9$ & 0.04 \\
\hline GGT & $151.3 \pm 138.0$ & $129.8 \pm 149.9$ & 0.11 \\
\hline SGPT & $308.6 \pm 630.9$ & $439.1 \pm 1093.0$ & 0.12 \\
\hline AP & $177.0 \pm 125.8$ & $165.7 \pm 135.7$ & 0.35 \\
\hline SGOT & $400.1 \pm 765.9$ & $695.8 \pm 1416.5$ & 0.17 \\
\hline R ratio & $9.6 \pm 23.8$ & $11.6 \pm 21.7$ & 0.34 \\
\hline
\end{tabular}

\section{Discussion}

Drug induced liver injury is the most under-recognized and under-reported cause of liver injury, ultimately leading to underestimation of its burden. The present study analyzes hospitalized patients suffering from drug induced liver injury who were admitted in a tertiary care center in Pakistan, over a seven-year period. This is a large data set related to DILI from a developing country from where there is paucity of such kind of information.

One important information that was revealed from this study is that the order and frequency of drugs associated with DILI is different from the list provided in the report from the Drug Induced Liver Injury Network (DILIN) and the Spanish Registry $[12,13]$. These studies showed that amoxicillin-clavulanate was the most common causative agent amongst the antimicrobials. A recently published review found 9 of the top 10 causes of DILI to be antimicrobial agents, majority of them antibiotics; this is a 
measure of their hepatotoxic potential, as well as the common use and duration of treatment with these drugs [20].

We found ATDs to be the most commonly implicated drug with approximately $64 \%$ of cases that were reviewed having received ATT. This reflects the differences in the epidemiology of infectious diseases and corresponded to numbers observed by other studies from this region [21]. ATDs was followed by homeopathic and herbal medications with $9 \%$ of cases having received it, similar to other prior studies [22]. After ATD, the category of drugs most frequently implicated in DILI were the homeopathic and herbal medications, with a frequency within a range provided in prior studies from regions with a history of common consumption [22]. More than $20 \%$ of patients in our series had encephalopathy accounting for fulminant or acute liver failure at the time of presentation in the hospital Conversely, the Spanish registry reported very low number of patients with fulminant hepatic failure with 11 out of 439 cases being classified as such $[13,23]$. The high prevalence of encephalopathy in our study can be attributed to a delay in presentation to the hospital with very little knowledge about the drug being a cause of liver injury. Additionally, our center is one of the main tertiary care hospitals in Pakistan that receives an increasing number of complicated referrals.

Another noteworthy observation deduced from our study is the fact that more than a quarter of hospitalized patients with DILI died while in the hospital. The mortality rate in our study appeared significantly high compared to that observed in several other studies, which ranges from 10 to $17.3 \%[9,12,24,25]$. This difference in mortality is perhaps due to the fact that our series of DILI is for hospitalized patients which are expected to be more severely ill. Another factor for high mortality in our study could be the fact that ATDs was the leading cause of DILI as it has been observed in an Indian study that mortality in DILI patients with ATDs was significantly high compared to those without ATDs; $21.5 \%$ vs. $11.4 \%$ respectively p $=0.02$ [26]. Lack of facilities for liver transplantation could be another reason for high mortality in our series. Very few studies have reported predictors of outcome for DILI the include hepatocellular damage, high bilirubin, female sex, as described by the US DILI network [14]. The Spanish registry and a Swedish study have described the hepatocellular pattern of damage as the most common form of liver injury associated with high incidence of liver transplantation or death if patient presented with jaundice $[13,25]$. In a Chinese study, ATDs were found to be the primary etiological factor for fatal DILI. Additionally, the same study also identified that hepatic encephalopathy, ascites, jaundice, alcohol abuse and direct bilirubin levels were associated with the death of DILI patients [27]. Likewise, in an Indian study, high-MELD score or a combination of ascites, encephalopathy, high bilirubin, prothrombin time, and leukocyte count were identified as predictors of mortality [26]. In our study, we also observed that mortality was significantly greater in patients with encephalopathy, male gender, hepatocellular pattern of DILI, increased INR ( $>1.5$ ) and patients on ventilatory support [28-35].

Limitations of the present study include a retrospective study design and a sample population based in a single tertiary care center setting. Non-availability of transplantation facility for ultimate treatment of patients restricted us from reviewing the outcomes in such patients in detail.

\section{Conclusion}

In the present study the most frequent cause of DILI in hospitalized patients was seen to be ATD. More than a quarter of patients died during hospital stay. As a result, care among physicians is required while prescribing potentially hepatotoxic agents. A close control of clinical and biochemical parameters are required while prescribing potentially hepatotoxic agents especially ATDs in our region. Additionally, efforts at the national level should be undertaken to make the public more aware about DILI especially while using ATDs.

\section{References}

1. Fontana RJ, Watkins PB, Bonkovsky HL, Chalasani N, Davern T, et al. (2009) Drug Induced Liver Injury N. Causes, clinical features, and outcomes from a prospective study of drug-induced liver injury in the United States. Gastroenterology 32(1): 55-68.

2. Seeff LB (2015) Drug-induced liver injury is a major risk for new drugs. J Dig Dis 33(4): 458-463.

3. Upadhyay AK, Kumar K, Kumar A, Mishra HS. (2010) Tinospora cordifolia (Willd.) Hook. f. and Thoms.(Guduchi)-validation of the Ayurvedic pharmacology through experimental and clinical studies. Int J Ayurveda Res 1(2): 112-121.

4. Porceddu M, Buron N, Roussel Cl, Labbe G, Fromenty B, et al. (2012) Prediction of liver injury induced by chemicals in human with a multiparametric assay on isolated mouse liver mitochondria. Toxicol Sci 129(2): 332-345.

5. Pandit A, Sachdeva T, Bafna P (2012) Drug-induced hepatotoxicity: a review. J Appl Pharm Sci 2(5): 233-243.

6. Ostapowicz G, Fontana RJ, Schiodt FV, Larson A, Davern TJ, et al. (2002) Results of a prospective study of acute liver failure at 17 tertiary care centers in the United States. Ann Intern Med 137(12): 947-954.

7. Agarwal VK, McHutchison JG, Hoofnagle JH (2010) Important elements for the diagnosis of drug-induced liver injury. Clin Gastroenterol Hepatol 8(5): 463-470.

8. Danan G, Benichou C (1993) Causality assessment of adverse reactions to drugs--I. A novel method based on the conclusions of international consensus meetings: application to drug-induced liver injuries. Clin Epidemiol 46(11): 1323-1330.

9. Bjornsson ES, Bergmann OM, Bjornsson HK, Kvaran RB, Olafsson $S$ (2013) Incidence, presentation, and outcomes in patients with drug-induced liver injury in the general population of Iceland. Gastroenterology 144(7): 1419-1425.

10. Sgro C, Clinard Fo, Ouazir K, Chanay H, Allard Cs, et al. (2002) Incidence of drug-induced hepatic injuries: a French population-based study. Hepatol 36(2): 451-455.

11. Ruigomez A, Brauer R, Rodrãguez LAG, Huerta C, Requena G, et al. (2014) Ascertainment of acute liver injury in two European primary care databases. Clin Pharmacol 70(10): 1227-1235. 
12. Chalasani N, Bonkovsky HL, Fontana R, Lee W, Stolz A, et al. (2015) Features and outcomes of 899 patients with drug-induced liver injury: the DILIN prospective study. Gastroenterology 148(7): 1340-1352.

13. Andrade RlJ, Lucena MI, FernÃ $\tilde{j}_{n d e z} \mathrm{MC}$, Pelaez G, Pachkoria $\mathrm{K}$, et al. (2005) Drug-induced liver injury: an analysis of 461 incidences submitted to the Spanish registry over a 10-year period. Gastroenterology 129(2): 512-521.

14. Navarro VJ, Barnhart H, Bonkovsky HL, Davern T, Fontana RJ (2014) Liver injury from herbals and dietary supplements in the U.S. DrugInduced Liver Injury Network. Hepatology 60(4): 1399-1408.

15. Bjornsson E, Jacobsen EI, Kalaitzakis E (2012) Hepatotoxicity associated with statins: reports of idiosyncratic liver injury postmarketing. J Hepatol 56(2): 374-380.

16. Charles EC, Olson KL, Sandhoff BG, McClure DL, Merenich JA (2005) Evaluation of cases of severe statin-related transaminitis within a large health maintenance organization. Am J Med 118(6): 618-624.

17. Kullak-Ublick GA, Andrade RJ, Merz M, End P, Benesic A, et al. (2017) Drug-induced liver injury: recent advances in diagnosis and risk assessment. Gut 66(6): 1154-1164.

18. Takeda M, Okamoto I, Nakagawa K (2015) Pooled safety analysis of EGFR-TKI treatment for EGFR mutation-positive non-small cell lung cancer. Lung Cancer 88(1): 74-79.

19. Yu YC, Mao YM, Chen CW, Chen JJ, Chen J, et al. (2017) CSH guidelines for the diagnosis and treatment of drug-induced liver injury. Hepatol Int 11(3): 221-241.

20. Hoofnagle JH, Björnsson ES (2019) Drug-Induced Liver Injury-Types and Phenotypes. N Engl J Med 381(3): 264-273.

21. Rathi C, Pipaliya N, Patel R, Ingle M, Phadke A, et al. (2017) Drug induced liver injury at a tertiary hospital in India: Etiology, clinical features and predictors of mortality. Annals of hepatol 16(3): 442-450.

22. Jing J, Teschke R (2018) Traditional Chinese medicine and herbinduced liver injury: comparison with drug-induced liver injury. J Clin Transl Hepatol 6(1): 57-68.

23. Suzuki A, Andrade RJ, Bjornsson E, Lucena MI, Lee WM, et al. (2010) Drugs associated with hepatotoxicity and their reporting frequency of liver adverse events in VigiBase: unified list based on international collaborative work. Drug safety 33(6): 503-522.
24. Chalasani NP, Hayashi PH, Bonkovsky HL, Navarro VJ, Lee WM, et al. (2014) ACG Clinical Guideline: the diagnosis and management of idiosyncratic drug-induced liver injury. Am J Gastroenterol 109(7): 950-966.

25. Bjornsson E, Olsson R (2005) Outcome and prognostic markers in severe drug-induced liver disease. Hepatol 42(2): 481-489.

26. Devarbhavi H, Dierkhising R, Kremers WK, Sandeep MS, Karanth D, et al. (2010) Single-center experience with drug-induced liver injury from India: causes, outcome, prognosis, and predictors of mortality. Am J Gastroenterol 105(11): 2396-2404.

27. Li B, Wang Z, Fang JJ, Xu CY, Chen WX (2007) Evaluation of prognostic markers in severe drug-induced liver disease. World J. Gastroenterol 13(4): 628-632.

28. Lucena MI, Andrade RIJ, Kaplowitz N, Garcia-Cortes M, Fernandez MC, et al. (2009) Phenotypic characterization of idiosyncratic drug-induced liver injury: the influence of age and sex. Hepatol 49(6): 2001-2009.

29. De Valle MB, Av Klinteberg V, Alem N, Olsson R, Bjornsson E (2006) Drug-induced liver injury in a Swedish University hospital out-patient hepatology clinic. Aliment Pharmacol Ther 24(8): 1187-1195.

30. Friis H, Andreasen PB (1992) Drug-induced hepatic injury: an analysis of 1100 cases reported to the Danish Committee on Adverse Drug Reactions between 1978 and 1987. J Intern Med 232(2): 133-138.

31. Almdal TP, Sorensen TI (1991) Incidence of parenchymal liver diseases in Denmark, 1981 to 1985: analysis of hospitalization registry data. Hepatol 13(4): 650-655.

32. Devarbhavi H, Dierkhising R, Kremers WK, Sandeep MS, Karanth D, et al. (2010) Single-center experience with drug-induced liver injury from India: causes, outcome, prognosis, and predictors of mortality. Am J Gastroenterol 105(11): 2396-2404.

33. Bies RR. Navarro VJ, Senior JR (2006) Drug-related hepatotoxicity. Journal of Clinical Pharmacology 46(9): 1052-1053.

34. Ibanez L, Perez E, Vidal X, Laporte JR (2002) Prospective surveillance of acute serious liver disease unrelated to infectious, obstructive, or metabolic diseases: epidemiological and clinical features, and exposure to drugs. Journal of hepatol 37(5): 592-600.

35. De Abajo FJ, Montero D, Madurga M, Garcia Rodriguez LA (2004) Acute and clinically relevant drug-induced liver injury: a population-based case-control study. Br J Clin Pharmacol 58(1): 71-80.

\section{Your next submission with JuniperPublishers will reach you the below assets}

- Quality Editorial service

- Swift Peer Review

- Reprints availability

- E-prints Service

- Manuscript Podcast for convenient understanding

- Global attainment for your research

- Manuscript accessibility in different formats

( Pdf, E-pub, Full Text, audio)

- Unceasing customer service

Track the below URL for one-step submission https://juniperpublishers.com/online-submission.php 